\title{
Ensuring the Safe and Effective Use of Medications During Pregnancy: Planning and Prevention Through Preconception Care
}

\author{
Janet D. Cragan · J. M. Friedman • Lewis B. Holmes • \\ Kathleen Uhl • Nancy S. Green • Laura Riley
}

Received: 17 November 2005 / Accepted: 20 April 2006 / Published online: 19 July 2006

(C) Springer Science+Business Media, Inc. 2006

Keywords Preconception care $\cdot$ Medications $\cdot$ Pregnancy Anticonvulsants $\cdot$ Asthma $\cdot$ Isotretinoin

\section{Introduction}

Whether to use a medication during pregnancy can be a concern for both women and health care providers. It has

The findings and conclusions in this report are those of the authors and do not necessarily represent the views of the Centers for Disease Control and Prevention.

J. D. Cragan $(\bowtie)$

National Center on Birth Defects and Developmental Disabilities, Centers for Disease Control and Prevention, MS E-86, 1600 Clifton Road, N.E., Atlanta, GA 30333

e-mail: jcragan@cdc.gov

J. M. Friedman

Department of Medical Genetics, University of British Columbia, Vancouver, British Columbia, Canada

\section{B. Holmes}

Genetics and Teratology Unit, MassGeneral Hospital for Children, Boston, MA

K. Uhl

Center for Drug Evaluation and Research, Food and Drug

Administration,

Silver Spring, MD

N. S. Green

March of Dimes Birth Defects Foundation,

White Plains, NY

\section{Riley}

Department of Obstetrics and Gynecology, Brigham and Women's Hospital,

Boston, MA been estimated that up to $10 \%$ of congenital anomalies may be caused by environmental exposures-that is, exposures to medications, alcohol, or other exogenous factors that have adverse effects on the developing embryo or fetus [1]. Avoiding all potentially adverse exposures can prevent these adverse effects. However, it is not always possible to avoid taking medications during pregnancy. Women who are pregnant continue to experience short-term and long-term health conditions that must be managed. Discontinuing treatment of a serious condition when a woman becomes pregnant can have profound, long-term implications both for her health and that of her baby. In a retrospective study from eight health maintenance organizations, researchers estimated that approximately 59\% of pregnant women were prescribed a medication other than a vitamin or mineral supplement at some time during pregnancy [2]. Use of over-the-counter medications during pregnancy may be even higher, and many women take a dietary or herbal supplement other than multivitamins or folic acid while pregnant $[3,4]$.

Preconception care provides the opportunity to optimize a woman's use of medications in preparation for pregnancy. Such care includes identifying patterns of medication use before pregnancy occurs; adjusting those patterns to avoid the use of nonessential medications; minimizing exposure to medications known to be harmful to the embryo or fetus; and adjusting the dose, route of administration, and timing of essential treatments to optimize maternal health at each stage of pregnancy while safeguarding the embryo, fetus, and infant. In this paper, we summarize the basic principles of teratology and the current state of knowledge about the effects of medication use during pregnancy; outline basic components of preconception care that can help minimize the risk of birth defects; and provide examples of approaches to planning for the safe and effective 
use of medications during pregnancy through preconception care.

\section{Principles of teratology}

The term "teratogen" is sometimes used to describe an agent that can produce structural or functional abnormalities in a developing embryo or fetus. Although this implies that an agent is inherently either teratogenic or not, teratogenicity is actually a property of the exposure taken as wholenot only the physical and chemical nature of the agent, but also the dose, route of administration, and timing in gestation, as well as concurrent exposure to other agents and biological susceptibility of the mother and embryo or fetus.

The developmental stage of the embryo or fetus at the time of exposure is critical. During conception and for about 2 weeks thereafter, most cells of the conceptus are not yet committed to a specific developmental program. One damaged cell can be replaced by another, and normal development will usually ensue, although the embryo will not survive if too many cells are damaged or killed. This is known as the "all-or-nothing" period, but even during this period some exposures can adversely affect the development of surviving embryos [5]. The subsequent period of organogenesis, from 18 to 60 days after conception (about 4.5-11 weeks after the start of the last normal menstrual period) is the time of greatest sensitivity to most teratogenic exposures. Fetal exposure later in gestation usually does not produce gross structural abnormalities, although there are exceptions. Adverse exposures during the fetal period more often result in growth restriction or functional disorders of the central nervous system, kidneys, or other organs.

Dose is a critical feature of any teratogenic exposure. Teratogenic effects occur only when the dose of an agent exceeds a certain threshold [1]. Medications that are generally considered safe during pregnancy can have adverse effects on the embryo or fetus if the mother takes them in doses that are so high that they cause maternal toxicity. Chronic exposure is usually of more concern than a single exposure, if the doses are similar. The route of exposure is also important. For example, risk is unlikely from the use of dermal agents that lack substantial systemic absorption. The teratogenicity of an exposure is also influenced by both the maternal and fetal genotypes, which can result in differences in cell sensitivity, placental transport, metabolism, receptor binding, or drug distribution. Some medications are metabolized extensively by the mother; their teratogenicity depends upon whether a toxic form reaches the embryo or fetus in sufficient quantities to produce adverse effects.

\section{Current state of knowledge about the effects of medication use during pregnancy}

\section{Maternal effects}

Many women begin pregnancy with medical conditions that require ongoing or episodic treatment. Examples include asthma, epilepsy, and hypertension. In addition, other medical problems, such as migraine headache or auto-immune disorders, may be exacerbated by pregnancy. When planning for the management of maternal conditions during pregnancy, it is important to distinguish conditions for which withholding treatment could be harmful to the mother, embryo, or fetus, from those for which cessation of treatment is unlikely to pose significant risk. For example, women with major depression who discontinue antidepressant medication before conception are at high risk of relapse and consequent self-injurious or even suicidal behavior [6]. In contrast, cessation of treatment for moderate hypercholesterolemia with a statin drug while a woman is pregnant is unlikely to increase her cardiovascular morbidity or mortality significantly.

Physiologic changes occur during pregnancy that can alter the effective dose of medications a woman is taking. Some changes occur abruptly, while others evolve slowly. Most begin during the first trimester and peak during the second trimester of pregnancy [7]. It may be necessary to adjust the dose and/or frequency of medication use repeatedly during pregnancy. Physiologic changes that can affect the pharmacokinetics and/or pharmacodynamics of medications during pregnancy include:

- Changes in total body weight and body fat composition.

- Delayed gastric emptying, prolonged gastrointestinal transit time, and decreased gastric acid secretion, all of which can affect the bioavailability of drugs [8-10].

- Expanded plasma volume and significantly increased extracellular fluid space and total body water content. These vary with the patient's weight and can affect the volume of distribution of drugs [11].

- Increased cardiac output, stroke volume, heart rate, and blood flow to the uterus, kidneys, skin, and mammary glands. The percentage of cardiac output attributed to hepatic blood flow is lower during pregnancy [12].

- Decreased concentration of plasma albumin, which can reduce the protein binding of some drugs [13].

- Increased glomerular filtration rate early in pregnancy, with a continued rise throughout pregnancy [14].

- Changes in the activity of hepatic enzymes, including the cytochrome P450 enzymes, xanthine oxidase, and Nacetyltransferase $[15,16]$.

Unfortunately, there are relatively few studies of drug pharmacokinetics during pregnancy. The dose of medications usually prescribed during pregnancy is the same 
used in nonpregnant adults, but this may result in substantial under- or over-dosage during pregnancy. When blood or serum concentrations of medications can be measured and the most effective level is known, they should be monitored throughout pregnancy and appropriate dosage adjustments made as needed. Further well-designed and well-conducted pharmacokinetic and pharmacodynamic studies of medications during pregnancy are needed.

Fetal effects

Maternal treatment with conventional doses of some medications during a susceptible period of pregnancy is known to be harmful to the developing embryo. Thalidomide and isotretinoin are the most notable examples, but there are others [17]. In contrast, taking some other medications or dietary supplements such as folic acid helps to prevent adverse pregnancy outcomes [18]. Because pregnant women are traditionally excluded from clinical trials for ethical reasons and because premarketing animal studies do not necessarily predict the effects of treatment in human pregnancy, little information about the teratogenic risks or safety of most drugs is available at the time they are marketed. Animal teratology studies are not routinely conducted for non-prescription drugs, vitamins, and dietary and herbal supplements, although these substances may produce pharmacological or toxic effects in the fetus. Moreover, there is no standard requirement for studies of adverse effects among children of women who took a drug during pregnancy after it has been approved by the FDA. In a review conducted in 2001, researchers found that there was not enough information to assess the teratogenic risk or safety during human pregnancy of more than $90 \%$ of prescription medications approved by the FDA in the previous 20 years [19].

Even when available, studies addressing fetal effects of maternal medication use during pregnancy may provide conflicting results or insufficient information to assess all potential outcomes or levels of risk. The concept of safety implies the absence of risk, which is impossible to demonstrate conclusively with any kind of study. Thus, it can be difficult for women and health care providers to decide whether to use a medication during pregnancy. The balance of risk, benefit, and efficacy of treatment for both mother and fetus is not always clear and must be individualized for different women under different circumstances.

\section{Basic components of preconception care that can minimize the risk of birth defects}

Serious congenital anomalies, including chromosome abnormalities and Mendelian disorders, can be identified in about
$2 \%$ of infants at birth [52]. However, some anomalies do not become apparent until later in life [20]. While most birth defects are not preventable, some can be avoided through appropriate planning and medical interventions. The following components of preconception care can help minimize the risk of birth defects:

- Optimize health before conception occurs. This includes counseling women to avoid smoking, use of excessive alcohol and illicit drugs, and exposure to potentially toxic environmental or occupational hazards before they are pregnant.

- Establish effective treatment for chronic conditions before conception occurs.

- Carefully manage all chronic conditions and intercurrent illnesses throughout pregnancy.

- Counsel women to avoid the use of nonessential medications, including prescription and over-the-counter medications and dietary or herbal supplements.

- Avoid the use of medications with high teratogenic risk when equally effective treatments with lower risks are available.

- Limit the use of essential medications to the smallest number of drugs possible that will effectively treat maternal disease without compromising the health of the woman or her fetus.

- Limit each essential medication to the smallest dose that can be used to effectively treat maternal disease without compromising the health of the woman or her fetus.

- Recommend that all women who are capable of becoming pregnant take a vitamin supplement or eat fortified foods to assure consumption of $0.4 \mathrm{mg}$ ( 400 micrograms) of folic acid per day.

Effective pregnancy management in women with chronic conditions requires careful planning, close medical supervision before and during pregnancy, and continuous communication between the pregnant woman and her health care providers.

\section{Examples of approaches to preconception planning for the use of medications during pregnancy in different clinical settings}

Exactly how the components of preconception planning for the use of medications during pregnancy are implemented depends on the nature of the condition requiring treatment, the known risks and safety of use of the specific drugs during pregnancy, and the woman's individual circumstances, among other factors. In this section, we provide three examples of approaches to planning for the safe and effective use of medications in clinical settings where these factors vary. 
Avoiding teratogenic treatments for non life-threatening maternal conditions-isotretinoin

Isotretinoin is indicated for the treatment of severe nodular cystic acne unresponsive to other therapy but is also used to treat non-nodular, but scarring, acne. A single course of therapy typically lasts $15-20$ weeks and can result in complete and prolonged remission of the acne in many patients. However, isotretinoin treatment in the first trimester of pregnancy is teratogenic. Exposed infants can have craniofacial, cardiac, thymic, and central nervous system malformations [21]. Research has also shown a high incidence of developmental delay in children whose mothers used isotretinoin early in the first trimester, regardless of whether the children had structural malformations [22].

Isotretinoin is indicated for use only in men and nonpregnant women. It should never be used during pregnancy. However, because approximately half of pregnancies in the United States are unintended, some women use isotretinoin in the early weeks of gestation before realizing they are pregnant [23]. Teratogenic outcomes have been reported after only one dose of isotretinoin during pregnancy [24]. The half-life of isotretinoin is approximately $24 \mathrm{~h}$, but about 2 weeks are required to eliminate $99 \%$ of the drug from the body after cessation of use.

Several risk management strategies have been implemented to prevent the use of isotretinoin during pregnancy, but pregnancy exposures continue to occur [25]. An enhanced risk management program called iPLEDGE became fully operational in March 2006 [26, 27]. iPLEDGE is a single, mandatory program for all marketed isotretinoin products. It requires that wholesalers, pharmacies, doctors, and patients register with the program in order to obtain the drug. Female patients who are capable of having children must obtain counseling about the risks of isotretinoin treatment and the requirements for its safe use; complete an informed consent form; have two negative pregnancy tests documented before starting isotretinoin therapy; use two different forms of contraception simultaneously or agree not to engage in heterosexual intercourse for one month prior to starting isotretinoin, throughout the course of treatment, and for one month after completing treatment; and have a negative pregnancy test documented every month during treatment and one month after completing treatment. More information about the iPLEDGE program can be found at www.ipledgeprogram.com.

Preconception care provides a unique and critical opportunity to prevent exposure to teratogens such as isotretinoin. Any woman who is considering pregnancy should be asked whether she is taking any medications including any preparation containing isotretinoin. If so, her health care provider should emphasize the risks of isotretinoin use during pregnancy, reevaluate the need for and duration of treatment, assess thoroughly the use and effectiveness of contraceptive measures, and reinforce the key elements of the iPLEDGE program. Women should be informed that conception cannot be attempted without risk of teratogenicity until one full month after the last dose of isotretinoin was taken. Educational information and materials from the iPLEDGE program can also be helpful in the setting of preconception care.

Managing maternal conditions that require continuous treatment—epilepsy

At least one in every 250 pregnant women, or about $0.4 \%$, takes an anticonvulsant drug [28]. Approximately half take the drugs to prevent seizures, but anticonvulsants are also used to manage mood disorders, migraine headaches, and chronic pain. Anticonvulsant drugs have several different modes of action, such as targeting a specific receptor or enzyme [29]. As a result, anticonvulsant medications vary in their effectiveness for specific types of epilepsy. Individuals with epilepsy may respond differently to particular medications, reflecting genetic differences such as polymorphisms in the cytochrome P450 enzymes. It is not yet possible to screen for pharmacogenetic differences that would help select the appropriate anticonvulsant for individual use. Physiologic changes during pregnancy also can affect the disposition of anticonvulsant drugs and the dose needed to prevent seizures [30].

While not all anticonvulsant drugs have been studied in pregnancy, a number of adverse effects have been identified in infants and children of women treated with these medications during pregnancy. Major malformations, midface and digit hypoplasia, microcephaly, growth restriction, and deficits in IQ are sometimes seen, although the pattern of abnormalities and specific effects vary for individual drugs $[28,31-33]$. For example, spina bifida occurs in approximately $1 \%$ of fetuses exposed to carbamazepine and in 2 to $5 \%$ of fetuses exposed to valproic acid $[34,35]$; microcephaly and growth restriction are observed more frequently in infants whose mothers took two or more anticonvulsant drugs simultaneously during pregnancy [28]; and subtle effects on intelligence have been identified in some studies of children exposed to carbamazepine, phenytoin, or phenobarbital in utero, although developmental delay and deficits in cognitive function are much more frequent with exposure to valproic acid [36-38]. While some infants exposed to an anticonvulsant drug in utero have abnormalities, others do not. Genetic differences in the fetal response to medications probably play a role.

It has been hypothesized that maternal epilepsy might cause fetal abnormalities independent of any drug effect, but several studies suggest that anticonvulsant treatments are usually responsible [39]. However, it seems likely that 
repeated or prolonged maternal seizures, such as occur in status epilepticus, can be devastating to the fetus [40]. Discontinuation or inadequate treatment of maternal epilepsy may be more dangerous to the fetus than effective anticonvulsant therapy.

Preconception care provides an opportunity to choose a plan of anticonvulsant treatment that will pose the least risk to the fetus while appropriately managing maternal symptoms. New data about the effects on the fetus of anticonvulsant medications are emerging steadily, and the latest information should always be sought. The treatment plan must be individualized for each woman in collaboration with her neurologist, psychiatrist, or other specialist. Primary considerations include assessing whether taking an anticonvulsant drug is essential to the mother's health; using the fewest number of anticonvulsant drugs possible; using the lowest dose for each drug that will effectively treat maternal symptoms [41]; establishing the most effective blood level of each drug before conception; and monitoring drug levels throughout pregnancy. For carbamazepine, phenobarbital, phenytoin, primidone, and valproic acid, levels of the non-protein bound, or "free," concentration should be measured. Because abrupt cessation of a medication may result in increased seizures or exacerbation of other symptoms, the number and dose of medications should be adjusted, and the woman's response stabilized, over a period of time before attempting conception.

In addition, preconception care offers the opportunity to consider whether additional measures, such as the use of a higher daily dose of folic acid, might be beneficial. Only limited and somewhat conflicting information is available about whether periconceptional supplementation with folic acid at levels higher than $0.4 \mathrm{mg}$ per day decreases the risk for neural tube defects associated with anticonvulsant drug exposure in utero [33, 42]. Currently, many women who require anticonvulsant drug therapy during pregnancy take 4 or $5 \mathrm{mg}$ of folic acid daily.

Managing maternal conditions with intermittent symptoms—asthma

Asthma is a chronic condition with intermittent symptoms, for which treatment during pregnancy is essential to safeguard the health and well-being of both the mother and fetus. Abrupt cessation or undertreatment of asthma during pregnancy can endanger both. Studies suggest that maternal asthma during pregnancy can increase the risk for perinatal mortality, preeclampsia, preterm delivery, and low birth weight $[43,44]$. Maternal asthma can lead to alkalosis with decreased blood flow to the uterus, decreased venous return, and a leftward shift of the oxyhemoglobin dissociation curve, all of which may contribute to fetal hypoxia. In the extreme, maternal hypoxia can result in decreased umbilical blood flow, increased systemic and pulmonary vascular resistance in the fetus, and decreased fetal cardiac output. Fetal compromise may occur well before maternal symptoms become severe [45]. Additional pathophysiologic mechanisms that could contribute to these adverse outcomes include hyperactivity of uterine and bronchial smooth muscle and the release of bioactive mediators during symptomatic asthma [43]. For these reasons, it is considered safer for pregnant women to be treated with asthma medications than to experience asthma symptoms and exacerbations [46].

A variety of medications are available to treat acute and chronic asthma. They include beta 2 -agonoists, corticosteroids, cromolyn, leukotriene modifiers, theophylline, and anticholinergics. These drugs have different mechanisms of action and thus potentially different effects on the fetus. Before attempting conception, it is important to maximize asthma control using medications that can also be used to manage asthma symptoms during pregnancy. This will ensure maximum oxygen delivery to the developing embryo and fetus while avoiding unintended medication exposures. A period of time may be needed to wean patients off some medications and to stabilize symptoms using others before attempting conception. In addition, because an estimated one third of pregnant women with asthma experience an increase in the frequency or severity of their symptoms during pregnancy, it is important to develop a plan before conception occurs for regular monitoring of asthma symptoms and pulmonary function during pregnancy and for early recognition and prompt treatment of exacerbations $[47,48]$. This plan should include a review of the proper use of medications, including the proper technique for using inhalers; education about self-monitoring and selfmanagement at home; and specific instructions about when to contact a health professional for additional care.

The National Asthma Education and Prevention Program Working Group outlines a step-wise approach for managing asthma during pregnancy in its report Managing Asthma During Pregnancy: Recommendations for Pharmaceutical Treatment-Update 2004. This report was developed after a systematic review of the current evidence from safety studies of asthma medications during pregnancy [46]. The approach is centered on the use of beta $a_{2}$-agonoists and corticosteroids, by inhalation when possible to minimize systemic absorption. The dose, frequency, and number of medications are decreased when possible but increased as needed, and pulmonary function is measured regularly (e.g., by monthly spirometry testing) during pregnancy. The approach also emphasizes the need for a treatment plan tailored to each patient's needs and circumstances, the frequency and severity of her symptoms, and her individual response to treatment. Regardless of the specifics, the benefits and risks of asthma treatment and of uncontrolled asthma during pregnancy for both the mother and fetus should be discussed 
during preconception planning so that a fully informed plan for asthma management can be agreed upon.

Additional measures can be instituted preconceptionally to minimize the severity or frequency of asthma symptoms during pregnancy, and thus decrease reliance on, and fetal exposure to, medications. Identifying, reducing, or eliminating exposure to allergens such as animal dander, housedust mites, cockroaches, pollen, and indoor mold; to irritants such as perfumes, sprays, and cleaning agents; and to smoke, including primary and secondhand tobacco smoke, wood burning stoves, or fireplaces, may decrease asthma symptoms. Preconception counseling also provides an opportunity to discuss the use of over-the-counter medications for symptoms such as rhinitis, congestion, and esophageal reflux that are often associated with asthma. While overthe-counter medications can be purchased without a prescription, they contain pharmacologically active ingredients that may affect the fetus. For example, studies have suggested that use of pseudoephedrine or aspirin in the first trimester may increase the risk of gastroschisis, a rare abdominal wall defect [49-51]. It has been theorized that gastroschisis could result from vascular disruption. Both aspirin and pseudoephedrine can have vasoactive effects, although the causal pathway has not been established for either drug, and the absolute risk of their use is very small. Most overthe-counter medications have not been adequately studied in pregnancy.

\section{Conclusion}

Although it is always better to avoid unnecessary medical treatment during pregnancy, some women with chronic conditions may not be able to become pregnant without appropriate therapy. In many other cases, proper treatment of a chronic condition during pregnancy may be safer for both the woman and her baby than stopping this treatment. It is important that women who are planning a pregnancy talk with their health care provider before beginning a new medication or making changes in current medications for the management of acute or chronic conditions. Continuous communication between a pregnant woman and her health care providers, careful preconceptional planning, effective management of conditions prior to pregnancy, and close medical supervision during pregnancy can help assure the best possible outcome for every woman and baby.

New information about the effects of medication use and the optimal management of maternal conditions during pregnancy continually becomes available. The following are regularly updated sources of such information and advice:

- The Organization of Teratology Information Specialists (OTIS) provides medical consultation, usually by phone, to individuals and health care providers about the reproductive risks of prenatal exposures.

Phone: 866-626-6847

Website: http://otispregnancy.org

- REPROTOX ${ }^{\circledR}$ is an information source for laboratory scientists, practicing physicians, and government agencies that contains commentaries on the effects of chemicals and physical agents on human pregnancy, reproduction, and development.

Website: www.reprotox.org

- The Teratogen Information System (TERIS) is a computerized database of summaries of individual agents designed to assist health care professionals in assessing the risks of exposures in pregnant women. Each summary is based on a review of the published literature and includes a risk assessment derived by consensus of an advisory board of authorities in clinical teratology.

Website: http://depts.washington.edu/ terisweb/teris

Information about the results of premarketing studies of individual medications, including animal reproductive studies and human clinical trials, is usually available from the drug manufacturer.

A list of many of the existing postmarketing pregnancy registries that seek to monitor exposure to specific medications during pregnancy is maintained by the Office of Women's Health, U.S. Food and Drug Administration at www.fda.gov/womens/registries.

Additional information is available from the March of Dimes at www.marchofdimes.com/pnhec/173_1453.asp.

\section{References}

1. Brent RL. Environmental causes of human congenital malformations: the pediatrician's role in dealing with these complex clinical problems caused by a multiplicity of environmental and genetic factors. Pediatrics 2004;113(4 Suppl):957-68.

2. Andrade SE, Gurwitz JH, Davis RL, Chan KA, Finkelstein JA, Fortman K, et al. Prescription drug use in pregnancy. Am J Obstet Gynecol 2004;191(2):398-407.

3. Werler MM, Mitchell AA, Hernandez-Diaz S, Honein MA. Use of over-the-counter medications during pregnancy. Am J Obstet Gynecol 2005;193:771-7.

4. Tsui B, Dennehy CE, Tsourounis C. A survey of dietary supplement use during pregnancy at an academic medical center. Am J Obstet Gynecol 2001;185(2):433-7.

5. Rutledge JC. Developmental toxicity induced during early stages of mammalian embryogenesis. Mutat Res 1997;396(1-2):113-27.

6. Nonacs R, Cohen LS. Assessment and treatment of depression during pregnancy: an update. Psychiatr Clin North Am 2003;26(3):547-62.

7. Uhl K. Conducting clinical pharmacology studies in pregnant and lactating women. In: Sahajwalla CG, (ed.). New Drug Development: Regulatory paradigms for clinical pharmacology and biopharmaceutics. New York: Marcel Dekker, Inc; 2004;267-96.

8. Hunt JN, Murray FA. Gastric function in pregnancy. J Obstet Gynaecol Br Emp 1958;65:78-83. 
9. Parry E, Shields R, Turnbull AC. Transit time in the small intestine in pregnancy. J Obstet Gynaecol Br Commonw 1970;77:900-1.

10. Gryboski WA, Spiro HM. The effect of pregnancy on gastric secretion. N Engl J Med 1976;155:1131-7.

11. Frederiksen MC, Ruo TI, Chow MJ, Atkinson AJ. Theophylline pharmacokinetics in pregnancy. Clin Pharmacol Ther 1986;40:321-8.

12. Robson SC, Mutch E, Boys RJ, Woodhouse KW. Apparent liver blood flow during pregnancy: a serial study using indocyanine green clearance. Brit J Obstet Gynaecol 1990;97:720-4.

13. Mendenhall HW. Serum protein concentrations in pregnancy: I. Concentrations in maternal serum. Am J Obstet Gynecol 1970;106:388-99.

14. Dunlop W. Serial changes in renal haemodynamics during normal human pregnancy. Br J Obstet Gynaecol 1981;88(1):1-9.

15. Tsutsumi K, Kotegawa T, Matsuki S, Tanaka Y, Ishii Y, Kodama $\mathrm{Y}$, et al. The effect of pregnancy on cytochrome P4501A2, xanthine oxidase, and $\mathrm{N}$-acetyltransferase activities in humans. Clin Pharmacol Ther 2001;70:121-5.

16. Wadelius M, Darj E, Frenne G, Rane A. Induction of CYP2D6 in pregnancy. Clin Pharmacol Ther 1997;62:400-7.

17. De Santis M, Straface G, Carducci B, Cavaliere AF, De Santis L, Lucchese A, et al. Risk of drug-induced congenital defects. Eur J Obstet Gynecol Reprod Biol 2004;117:10-19.

18. CDC. Recommendations for the use of folic acid to reduce the number of cases of spina bifida and other neural tube defects. MMWR Morb Mortal Wkly Rep 1992;41(RR-14):1-7.

19. Lo WY, Friedman JM. Teratogenicity of recently introduced medications in human pregnancy. Obstet Gynecol 2002;100(3):46573.

20. Baird PA, Anderson TW, Newcombe HB, Lowry RB. Genetic disorders in children and young adults: a population study. Am J Hum Genet 1988;42(5):677-93.

21. Lammer EJ, Chen DT, Hoar RM, Agnish ND, Benke PJ, Braum JT, et al. Retinoic acid embryopathy. N Engl J Med 1985;313:83741.

22. Adams J, Lammar EJ. Neurobehavioral teratology of isotretinoin. Reprod Toxicol 1993;7(2):175-7.

23. Henshaw SK. Unintended pregnancy in the United States. Fam Plann Perspect 1998;30(1):24-9, 46.

24. Department of Health and Human Services. Update on birth defects with isotretinoin. FDA Drug Bulletin 1984;14:15-6.

25. Honein MA, Paulozzi LJ, Erickson JD. Continued occurrence of Accutane ${ }^{\circledR}$-exposed pregnancies. Teratology 2001;64(3):142-7.

26. FDA Center for Drug Evaluation Research. [homepage on the Internet]. Washington (DC): FDA; [created 2005 Aug 12; cited 2005 Oct 19]. FDA Public Health Advisory. Strengthened risk management program for isotretinoin. Available from: http://www.fda.gov/cder/drug/advisory/isotretinoin2005.htm.

27. FDA Center for Drug Evaluation Research. [homepage on the Internet]. Washington (DC): FDA; [created 2005 Aug 12; cited 2005 Oct 19]. Accutane (isotretinoin) Questions and Answers. Available from: http://www.fda.gov/cder/drug/infopage/accutane/accutane_QA20050812.htm.

28. Holmes LB, Harvey EA, Coull BA, Huntington BA, Khoshbin S, Hayes AM, et al. The teratogenicity of anticonvulsant drugs. $\mathrm{N}$ Engl J Med 2001;344:1132-8.

29. Holmes GL. The interface of preclinical evaluation with clinical testing of antiepileptic drugs: role of pharmacogenomics and pharmacogenetics. Epilepsy Res 2002;50:41-54.

30. Pennell PB. Antiepileptic drug pharmacokinetics during pregnancy and lactation. Neurology 2003;61:S35-42.

31. Morrow JI, Russell A, Guthrie E, Parsons L, Robertson I, Waddell $\mathrm{R}$, et al. Malformation risks of antiepileptic drugs in pregnancy: a prospective study from the UK Epilepsy and Pregnancy Register. J Neurol Neurosurg Psychiatry In press 2005.
32. Holmes LB, Wyszynski DF, Lieberman E. The AED (antiepileptic drug) Pregnancy Registry: a 6-year experience. Arch Neurol 2004;61:673-8.

33. Wyszynski D, Nambisan M, Surve T, Alsdorf RM, Smith C, Holmes LB. Increased risk of major malformations in offspring exposed to valproate during pregnancy. Neurology 2005;64: 961-5.

34. Rosa FW. Spina bifida in infants of women treated with carbamazepine during pregnancy. N Engl J Med 1991;324:674-7.

35. Omtzigt JGC, Los JF, Grobbee DE, Pijpers L, Jahoda MGJ, Brandenburg $\mathrm{H}$, et al. The risk of spina bifida aperta after firsttrimester exposure to valproate in a prenatal cohort. Neurology 1992;42(5 Suppl):119-25.

36. Scolnik D, Nulman I, Rovet J, Gladstone D, Czuchta D, Gardner HA, et al. Neurodevelopment of children exposed in utero to phenytoin and carbamazepine monotherapy. JAMA 1994;271:767-70.

37. Reomoscj KM, Sanders SA, Rubin DB. In utero exposure to phenobarbital and intelligence deficits in adult men. JAMA 1995; 724:1518-25.

38. Ahab N, Kini U, Vinten J, Ayres J, Baker G, Clayton-Smith J, et al. The longer term outcome of children born to mothers with epilepsy. J Neurol Neurosurg Psychiatry 2004;75:1575-83.

39. Fried S, Kozer E, Nulman I, Einarson TR, Koren G. Malformation rates in children of women with untreated epilepsy: a metaanalysis. Drug Saf 2004;27:197-202.

40. Minkoff H, Schaffer RM, Delke I, Grunebaum AN. Diagnosis of intracranial hemorrhage in utero after a maternal seizure. Obstet Gynecol 1985;65(Suppl):22S-24S.

41. Mawer G, Clayton-Smith J, Coyle H, Kini U. Outcome of pregnancy in women attending an outpatient epilepsy clinic: adverse features associated with higher doses of sodium valproate. Seizure 2002;11:512-8.

42. Hernández-Díaz S, Werler MM, Walker AM, Mitchell AA. Neural tube defects in relation to use of folic acid antagonists during pregnancy. Am J Epidemiol 2001;153:961-8.

43. Demissie K, Breckenridge MB, Rhoads GG. Infant and maternal outcomes in the pregnancies of asthmatic women. Am J Respir Crit Care Med 1998;158:1091-5.

44. Källén B, Rydhstroem H, Åberg A. Asthma during pregnancy-a population based study. Eur J Epidemiol 2000;16:167-71.

45. Cunningham FG, Gant NF, Leveno KJ, Gilstrap LC, Hauth JC, Wenstrom KD. Williams obstetrics. 21st ed. New York: McGrawHill; 2001.

46. The National Asthma Education and Prevention Program Working Group. Quick Reference. Managing Asthma During Pregnancy: Recommendations for Pharmaceutical Treatment-Update 2004. Bethesda, MD: National Institutes of Health: National Heart, Lung, and Blood Institute; 2004.

47. Schatz M, Dombrowski MP, Wise R, Thom EA, Landon M, Mabie $\mathrm{W}$, et al. Asthma morbidity during pregnancy can be predicted by severity classification. J Allergy Clin Immunol 2003;112: 283-8.

48. Kwon HL, Belanger K, Bracken MB. Effect of pregnancy and stage of pregnancy on asthma severity: a systematic review. Am J Obstet Gynecol 2004;190:1201-10.

49. Torfs CP, Katz EA, Bateson TF, Lam PK, Curry CJR. Maternal medications and environmental exposures as risk factors for gastroschisis. Teratology 1996;54:84-92.

50. Martínez-Frías ML, Rodríguez-Pinilla E, Prieto L. Prenatal exposure to salicylates and gastroschisis: a case-control study. Teratology 1997;56:241-3.

51. Werler MM, Sheehan JE, Mitchell AA. Maternal medication use and risks of gastroschisis and small intestinal atresia. Am J Epidemiol 2002;155:26-31.

52. Nelson K, Holmes LB. Malformations due to presumed spontaneous mutations in newborn infants. N Engl J Med 1989;320: 19-23. 